\title{
Diversity of yam bean (Pachyrhizus spp. Fabaceae) based on morphoagronomic traits in the Brazilian Amazon
}

\author{
Edinei Santos da SILVA ${ }^{1}$, Danilo Fernandes da SILVA FILHO², César Augusto TICONA-BENAVENTE2 \\ 1 Universidade Federal do Amazonas, Postgraduate Program in Tropical Agriculture, Manaus, CEP: 69077-000, Brazil \\ 2 Instituto Nacional de Pesquisas da Amazônia, Plant Breeding Laboratory, Manaus, CEP: 69067-375 Brazil \\ * Corresponding author: cesar.benavente@gmail.com
}

\section{ABSTRACT}

Yam bean is a non-conventional horticultural crop adapted to the Amazon region. It presents edible roots, yet, its seeds contain cytotoxic components such as rotenone and pachyrhizin. The Instituto Nacional de Pesquisas da Amazonia has 64 yam bean genotypes in its germplasm bank, however, their diversity is unknown. The aim of this study was to assess the diversity of these genotypes using 10 morphoagronomic traits, plant height, number of secondary branches, shoot biomass, stem diameter, root biomass, number, length, diameter, length/diameter ratio (L/D), and shape. The accessions were planted on non-flooded land, Manaus, Amazonas (02'59'48.2”S and 60'01'22.4”W) in completely randomized design with three replicates and three plants per plot spaced $0.5 \times 1 \mathrm{~m}$. The results showed significant diversity for all characters except for stem diameter and visual assessment of root shape. Biplot graphic explained $60 \%$ of the total variation, which identified that genotypes P44, P22 and P18 have high values for root yield (80-108 $\left.\mathrm{t} \mathrm{ha}^{-1}\right)$, number of secondary branches (15-31) and shoot biomass (0.5-0.8 $\left.\mathrm{kg} \mathrm{plant}^{-1}\right)$. Cluster analysis, considering $50 \%$ of relative Euclidean distance, revealed 15 major groups. We conclude that the genotypes assessed have wide diversity and some of them high root yield potential. This suggests that a yam bean breeding program can be successful for the yield of roots and seeds in the Amazonian region.

KEYWORDS: Feijão-macuco, Jacatupé, biodiversity, Amazon region.

\section{Diversidade de feijão-macuco (Pachyrhizus spp. Fabaceae) baseada em caracteres morfoagronômicos na amazônia brasileira}

\section{RESUMO}

O feijão-macuco é uma hortaliça não convencional da Amazônia. Suas raízes são comestíveis e suas sementes tóxicas por conter rotenona e paquirizina. O Instituto Nacional de Pesquisas da Amazônia (INPA) tem conservado 64 genótipos de feijão-macuco em seu banco de germoplasma, mas a diversidade genética destes é desconhecida. O objetivo deste trabalho foi avaliar estes acessos utilizando 10 descritores morfoagronômicos tais como: altura de planta, número de ramas secundárias, massa da parte aérea, diâmetro de caule, massa, número, comprimento, diâmetro, relação comprimento/diâmetro e formato de raízes. O experimento foi conduzido em terra firme no município de Manaus (02 59'48.2"LS e 60 01' 22.4”LO), em delineamento de blocos ao acaso com três repetiçóes e três plantas por parcela. Os resultados mostraram diversidade significativa para todos os caracteres, exceto para diâmetro do caule e avaliação visual do formato da raiz. O Biplot explicou $60 \%$ da variação total o qual mostrou que os genótipos P44, P22 e P18 tem elevada produtividade de raízes (80-108 t ha $\left.{ }^{-1}\right)$, número de ramificaçóes secundarias (15-31) e massa da parte aérea (0.5-0.8 kg/planta). A análise de agrupamento considerando 50\% da distancia euclidiana relativa mostrou 15 grupos principais. Por tanto, concluímos haver ampla diversidade nos genótipos especialmente para produtividade de raízes. Isto indica que o melhoramento de feijáo-macuco, na regiáo Amazônica, pode ter sucesso para aumentar a produtividade de raízes e sementes.

PALAVRAS-CHAVE: Feijão-macuco, Jacatupé, biodiversidade, Amazônia. 


\section{INTRODUCTION}

Conserving germplasm of yam bean (Pachyrhizus spp.) is necessary, since it is a species well adapted to the poor soils of the Amazonian and Andean regions. It is still not an economically important species in these regions although, it can produce up to $125.9 \mathrm{t} \mathrm{ha}^{-1}$ root yield (Nielsen et al. 2000; Tapia and Sorensen 2003). Its roots are tuberous and edible, either fresh or cooked (Kinupp and Lorenzi 2014). Their flesh and periderm are white and brown, respectively. These roots are attractive due to their nutritional value due to their high protein, as well as starch (Padonou et al. 2013), iron (Chavez et al. 2000) and zinc (Heider et al. 2011) content. On account of these relevant characteristics, they have been introduced into West Africa to help mitigate hunger (Zanklan et al. 2007). Genetically, these species are autogamous, with outcrossing ratios ranging between 2 and $4 \%$ (Sørensen 1996).

On the other hand, seeds bear a high content rotenone and pachyrhizin which are toxic molecules (Estrella-Parra et al. 2014; Lautié et al. 2013; Leuner et al. 2013). For this reason, they can be used as pesticides and piscicides, or as food if seeds were treated with temperature or chemicals to degrade the rotenone stability (Catteau et al. 2013). Yam bean is composed by three cultivated species (P. erosus, $P$. ahipa and $P$. tuberosus) and two wild (P. ferrugineous and P. panamensis). The first three come from the Amazon, Mexico and subtropical east Andeans (Bolivia and Argentina), respectively.

The Instituto Nacional de Pesquisas da Amazônia (INPA), located in Manaus, Amazonas, Brazil, has conserved some yam bean accessions for over 30 years. Initially, these materials were introduced from Mexico (P. erosus) and Mato GrossoBrazil (P. tuberosus). However, natural outcrossing led to increase the diversity, which generated 64 highly endogamous genotypes. Probably, it is the major collection from Brazil and the Amazonian region.

In these genotypes root yield and characteristics related of seeds and roots have been assessed (Ribeiro et al. 2011). However, their genetic diversity was not studied.

Two approaches have been used in this species to evaluate morphological (Tapia and Sorensen 2003) and molecular diversity (Delêtre et al. 2013; Santayana et al. 2014). As our interest is to start a yam bean breeding program in the Brazilian Amazon, the morphoagronomic approach is more useful. The aim of this study was to assess the yam bean diversity of the INPA germplasm bank using 10 morphoagronomic traits.

\section{MATERIALS AND METHODS}

Plant materials consisted of 64 yam bean genotypes from the INPA germplasm bank, which were obtained through selection from other accessions introduced from México (P. erosus) and Mato Grosso State, Brazil (P. tuberosus) in the 1980s. Presently, the yam bean genotypes'taxonomic denominations are unknown since natural outcrossings led to species identity loss.

The experiment was conducted at the vegetable field of INPA (02० 59'48.2"S and 60 01' 22.4”W), during January to August, 2014. In Manaus, historically, the mean annual rainfall has been $2567 \mathrm{~mm}$ (mainly from November to June) and mean temperature $31.4^{\circ} \mathrm{C}$ (Antonio 2006). The soil used was non-flooded land, red-yellow Ultisoil, sandy texture and $\mathrm{pH}=6.0$. This soil type is poor in organic material; therefore, it was fertilized using $2 \mathrm{~kg} \mathrm{~m}^{-2}$ of organic compost.

The accessions were planted in completely randomized design, with three replications and three plants per plot. The spacing was $1 \times 0.5 \mathrm{~m}$ between rows and plants respectively. The measured traits were plant height $(\mathrm{cm})$, number of secondary branches, shoot biomass $\left(\mathrm{kg}\right.$ plant $\left.{ }^{-1}\right)$, stem diameter $(\mathrm{mm})$, root yield $\left(\mathrm{kg} \mathrm{plant}^{-1}\right)$, length $(\mathrm{mm})$, diameter $(\mathrm{mm})$, length/diameter ratio and shape (1=globular, $2=$ elongated, $3=$ more elongated). These traits are important from an agronomic point of view.

Flowers were pruned weekly during four months since 100 days after planting, when $50 \%$ of experimental area was flowering. This practice may enhance root yield (Noda and Kerr 1983). The roots were harvested in the seventh month after planting.

Data were analyzed by analysis of variance (ANOVA) and Duncan tests $(\mathrm{P} \leq 0.05)$. In addition, with standardized data we made a Biplot Graphic using R (R Core Team 2015), package GGEBiplotGUI (Bernal and Villardon 2015). This analysis shows the relation between characters, accessions and the interaction of characters $\mathrm{x}$ accessions. The distances between genotypes were estimated through Euclidean distance by using standardized values and performing 1000 bootstraps. Then, the accessions were grouped by UPGMA method (Unweighted pair group method with arithmetic mean) and presented in a dendrogram using Darwin 6.0.8 software (Perrier and Jacquemoud-Collet 2006).

\section{RESULTS}

To start a yam bean breeding program it is necessary to have information about diversity, especially for agronomical characters. ANOVA's and Duncan tests showed that there is significant variability for most of the characters (Table 1). Only stem diameter and root shape assessed visually, showed non-significant variability by the $\mathrm{F}$ test $(\mathrm{P} \geq 0.05)$. Hence, these two characters are useless to characterize diversity.

Biplot analysis (Figure 1) explained $60 \%$ of total variability; the wide distribution of genotypes in the graphic shows diversity of yam bean and the power of morphoagronomic traits to detect diversity. To find the highest yielding accessions 
Table 1. Means for 10 traits in 64 yam bean genotypes (Pachyrhizus spp.). Manaus, 2014.

\begin{tabular}{|c|c|c|c|c|c|c|c|c|c|c|}
\hline Genotype & $\begin{array}{l}\text { Stem } \\
\text { diameter }^{1} \\
(\mathrm{~mm})\end{array}$ & $\begin{array}{l}\text { Plant } \\
\text { height } \\
(\mathrm{m})\end{array}$ & $\begin{array}{l}\text { Secondary } \\
\text { branches } \\
\text { number }\end{array}$ & $\begin{array}{l}\text { Shoot } \\
\text { biomass per } \\
\text { plant } \\
(\mathrm{kg}) \\
\end{array}$ & $\begin{array}{l}\text { Root } \\
\text { yield per plant } \\
(\mathrm{kg})\end{array}$ & $\begin{array}{l}\text { Number of } \\
\text { roots per } \\
\text { plant }\end{array}$ & $\begin{array}{l}\text { Root } \\
\text { diameter (D) } \\
\text { (cm) }\end{array}$ & $\begin{array}{l}\text { Root } \\
\text { length }(\mathrm{L}) \\
(\mathrm{cm})\end{array}$ & L/D ratio & $\begin{array}{l}\text { Root } \\
\text { shape }{ }^{1,2}\end{array}$ \\
\hline P1 & 9.33 & $0.73 a b$ & $15.33 \mathrm{abc}$ & $0.31 \mathrm{abcd}$ & $3.74 a b$ & $2.67 \mathrm{ab}$ & $16.0 \mathrm{abc}$ & $16.00 a b$ & $1.00 \mathrm{~b}$ & 3.00 \\
\hline P2 & 9.33 & $0.77 a b$ & $11.67 \mathrm{bc}$ & $0.25 \mathrm{abcd}$ & $3.12 a b$ & $2.67 a b$ & $15.67 \mathrm{abc}$ & $13.33 a b$ & $0.90 \mathrm{~b}$ & 3.00 \\
\hline P3 & 7.67 & $0.99 a b$ & $12.00 \mathrm{bc}$ & $0.50 \mathrm{abcd}$ & $3.50 a b$ & $2.33 \mathrm{ab}$ & $20.67 a b$ & $15.00 a b$ & $0.73 \mathrm{~b}$ & 1.67 \\
\hline P4 & 8.00 & $0.64 a b$ & $10.00 \mathrm{bc}$ & 0.17 abcd & $2.53 \mathrm{ab}$ & $2.67 \mathrm{ab}$ & $16.00 \mathrm{abc}$ & $15.67 \mathrm{ab}$ & $0.97 \mathrm{~b}$ & 3.00 \\
\hline P5 & 8.33 & $0.84 a b$ & $16.33 \mathrm{abc}$ & $0.23 \mathrm{abcd}$ & $3.02 a b$ & $1.67 \mathrm{~b}$ & $20.00 a b$ & $14.00 \mathrm{ab}$ & $0.70 \mathrm{~b}$ & 1.67 \\
\hline P6 & 7.67 & $1.05 a b$ & $14.67 \mathrm{abc}$ & $0.29 \mathrm{abcd}$ & $4.40 a b$ & $2.67 \mathrm{ab}$ & $16.33 \mathrm{abc}$ & $17.00 a b$ & $1.12 b$ & 3.00 \\
\hline P7 & 6.00 & $0.94 \mathrm{ab}$ & $14.00 \mathrm{abc}$ & $0.25 \mathrm{abcd}$ & $3.39 a b$ & $2.67 a b$ & $17.00 \mathrm{abc}$ & $12.00 \mathrm{ab}$ & $0.69 \mathrm{~b}$ & 3.00 \\
\hline P8 & 5.00 & $0.84 a b$ & $15.00 \mathrm{abc}$ & $0.13 \mathrm{~cd}$ & $2.62 \mathrm{ab}$ & $2.67 \mathrm{ab}$ & $13.00 \mathrm{abc}$ & $14.33 \mathrm{ab}$ & $1.16 \mathrm{~b}$ & 3.00 \\
\hline P9 & 7.67 & $0.61 \mathrm{ab}$ & $12.67 \mathrm{bc}$ & $0.09 \mathrm{~d}$ & $2.16 \mathrm{ab}$ & $1.33 b$ & $18.33 a b c$ & $13.33 a b$ & $0.79 \mathrm{~b}$ & 2.33 \\
\hline P10 & 6.00 & $0.83 a b$ & $13.67 \mathrm{abc}$ & $0.30 \mathrm{abcd}$ & $1.97 \mathrm{ab}$ & $2.33 \mathrm{ab}$ & $14.33 \mathrm{abc}$ & $12.00 a b$ & $0.83 \mathrm{~b}$ & 3.00 \\
\hline P11 & 5.00 & $0.67 \mathrm{ab}$ & $9.33 \mathrm{bc}$ & $0.07 \mathrm{~d}$ & $1.55 b$ & $2.67 \mathrm{ab}$ & $12.33 \mathrm{abc}$ & $11.67 \mathrm{ab}$ & $0.99 \mathrm{~b}$ & 2.33 \\
\hline P12 & 4.67 & $1.42 \mathrm{ab}$ & $14.67 \mathrm{abc}$ & $0.42 \mathrm{abcd}$ & $1.57 \mathrm{~b}$ & $3.33 \mathrm{ab}$ & $11.67 \mathrm{bc}$ & $10.00 \mathrm{~b}$ & $0.89 \mathrm{~b}$ & 3.00 \\
\hline P13 & 8.00 & $0.74 a b$ & 10.67 bc & $0.48 \mathrm{abcd}$ & $1.94 \mathrm{ab}$ & $1.67 \mathrm{~b}$ & $14.67 \mathrm{abc}$ & $16.67 a b$ & $1.22 \mathrm{~b}$ & 2.00 \\
\hline P14 & 5.00 & $1.18 \mathrm{ab}$ & $13.33 \mathrm{abc}$ & $0.30 \mathrm{abcd}$ & $2.67 \mathrm{ab}$ & $2.67 \mathrm{ab}$ & $16.00 \mathrm{abc}$ & $9.67 \mathrm{~b}$ & $0.73 \mathrm{~b}$ & 2.33 \\
\hline P15 & 6.67 & $0.58 a b$ & $12.00 \mathrm{bc}$ & $0.24 \mathrm{abcd}$ & $2.25 a b$ & $2.00 \mathrm{ab}$ & $13.33 \mathrm{abc}$ & $9.33 \mathrm{~b}$ & $0.73 \mathrm{~b}$ & 3.00 \\
\hline P16 & 4.33 & $1.00 \mathrm{ab}$ & $20.67 \mathrm{abc}$ & $0.36 \mathrm{abcd}$ & $2.00 \mathrm{ab}$ & $2.00 \mathrm{ab}$ & $13.33 \mathrm{abc}$ & $10.67 \mathrm{~b}$ & $0.83 \mathrm{~b}$ & 1.67 \\
\hline P17 & 7.67 & $1.35 a b$ & $18.00 \mathrm{abc}$ & $0.56 \mathrm{abcd}$ & $4.17 \mathrm{ab}$ & $2.00 a b$ & $20.00 a b$ & $10.33 \mathrm{~b}$ & $0.52 \mathrm{~b}$ & 2.33 \\
\hline P18 & 7.33 & $1.14 a b$ & $23.00 \mathrm{abc}$ & $0.54 \mathrm{abcd}$ & $4.28 \mathrm{ab}$ & $1.33 \mathrm{~b}$ & $22.00 \mathrm{a}$ & $23.33 \mathrm{a}$ & $1.06 \mathrm{~b}$ & 1.33 \\
\hline P19 & 9.00 & $0.93 a b$ & 10.67 bc & $0.25 \mathrm{abcd}$ & $2.92 \mathrm{ab}$ & $2.00 \mathrm{ab}$ & $18.33 \mathrm{abc}$ & $12.00 \mathrm{ab}$ & $0.64 \mathrm{~b}$ & 3.00 \\
\hline P20 & 6.67 & $1.17 \mathrm{ab}$ & $15.67 \mathrm{abc}$ & $0.32 \mathrm{abcd}$ & $3.32 \mathrm{ab}$ & $3.67 \mathrm{ab}$ & $15.33 \mathrm{abc}$ & $9.33 \mathrm{~b}$ & $0.59 \mathrm{~b}$ & 1.67 \\
\hline P21 & 7.67 & $0.95 a b$ & $15.67 \mathrm{abc}$ & $0.57 \mathrm{abcd}$ & $3.49 a b$ & $2.67 \mathrm{ab}$ & $19.00 \mathrm{abc}$ & $14.67 \mathrm{ab}$ & $0.80 \mathrm{~b}$ & 2.33 \\
\hline P22 & 10.33 & $1.32 \mathrm{ab}$ & $15.00 \mathrm{abc}$ & $0.82 \mathrm{a}$ & $5.38 \mathrm{a}$ & $3.33 a b$ & $19.67 \mathrm{abc}$ & $14.67 \mathrm{ab}$ & $0.74 \mathrm{~b}$ & 2.33 \\
\hline P23 & 7.00 & $0.86 \mathrm{ab}$ & $20.00 a b c$ & $0.56 \mathrm{abcd}$ & $3.36 \mathrm{ab}$ & $3.00 a b$ & $16.67 \mathrm{abc}$ & $15.00 \mathrm{ab}$ & $0.92 \mathrm{~b}$ & 1.67 \\
\hline P24 & 7.00 & $0.70 a b$ & 11.33 bc & $0.23 \mathrm{abcd}$ & $1.92 \mathrm{ab}$ & $3.00 \mathrm{ab}$ & 12.33abc & $17.33 a b$ & $1.37 \mathrm{~b}$ & 2.67 \\
\hline P25 & 6.67 & $0.72 a b$ & $13.33 a b c$ & $0.29 \mathrm{abcd}$ & $2.14 a b$ & $2.00 a b$ & $16.00 \mathrm{abc}$ & $13.33 a b$ & $0.83 \mathrm{~b}$ & 2.33 \\
\hline P26 & 7.33 & $0.77 \mathrm{ab}$ & $18.33 \mathrm{abc}$ & $0.59 \mathrm{abcd}$ & $3.46 a b$ & $3.33 a b$ & $16.33 \mathrm{abc}$ & $16.00 a b$ & $4.19 \mathrm{a}$ & 2.33 \\
\hline P27 & 6.67 & $0.85 a b$ & $16.33 \mathrm{abc}$ & $0.47 \mathrm{abcd}$ & $3.31 \mathrm{ab}$ & $2.67 \mathrm{ab}$ & $17.67 \mathrm{abc}$ & $11.67 a b$ & $0.66 \mathrm{~b}$ & 3.00 \\
\hline P28 & 8.33 & $0.88 \mathrm{ab}$ & $14.67 \mathrm{abc}$ & $0.35 \mathrm{abcd}$ & $3.55 a b$ & $2.67 \mathrm{ab}$ & $18.33 \mathrm{abc}$ & $11.67 a b$ & $0.64 \mathrm{~b}$ & 3.00 \\
\hline P29 & 8.67 & $0.66 \mathrm{ab}$ & $12.67 \mathrm{bc}$ & $0.24 \mathrm{abcd}$ & $3.18 \mathrm{ab}$ & $2.00 a b$ & $18.00 \mathrm{abc}$ & $12.67 a b$ & $0.71 \mathrm{~b}$ & 1.67 \\
\hline P30 & 7.00 & $0.49 \mathrm{~b}$ & $11.67 \mathrm{bc}$ & $0.31 \mathrm{abcd}$ & $3.43 a b$ & $2.67 a b$ & $17.67 \mathrm{abc}$ & $10.33 b$ & $0.60 \mathrm{~b}$ & 1.67 \\
\hline P31 & 6.00 & $0.74 \mathrm{ab}$ & 11.67 bc & $0.09 \mathrm{~d}$ & $2.02 a b$ & $2.33 \mathrm{ab}$ & $13.67 \mathrm{abc}$ & $16.67 \mathrm{ab}$ & $1.33 \mathrm{~b}$ & 2.33 \\
\hline P32 & 6.33 & $0.99 \mathrm{ab}$ & $14.33 a b c$ & $0.21 \mathrm{abcd}$ & $1.54 \mathrm{~b}$ & $2.00 \mathrm{ab}$ & $13.33 \mathrm{abc}$ & $11.67 a b$ & $1.12 \mathrm{~b}$ & 1.67 \\
\hline P33 & 7.33 & $1.02 \mathrm{ab}$ & $19.67 \mathrm{abc}$ & $0.21 \mathrm{abcd}$ & $2.36 \mathrm{ab}$ & $3.00 \mathrm{ab}$ & $14.67 \mathrm{abc}$ & $11.33 \mathrm{~b}$ & $0.84 \mathrm{~b}$ & 3.00 \\
\hline P34 & 8.33 & $0.96 \mathrm{ab}$ & $16.33 a b c$ & $0.32 \mathrm{abcd}$ & $2.92 \mathrm{ab}$ & $3.00 a b$ & $13.67 \mathrm{abc}$ & $15.00 \mathrm{ab}$ & $1.14 b$ & 3.00 \\
\hline P35 & 7.33 & $0.47 \mathrm{~b}$ & $10.00 \mathrm{bc}$ & $0.17 \mathrm{abcd}$ & $1.83 \mathrm{ab}$ & $2.67 \mathrm{ab}$ & $14.67 \mathrm{abc}$ & $17.00 \mathrm{ab}$ & $1.15 b$ & 2.33 \\
\hline P36 & 6.67 & $0.58 \mathrm{ab}$ & $12.67 \mathrm{bc}$ & $0.11 d$ & $1.34 b$ & $2.67 \mathrm{ab}$ & $13.00 \mathrm{abc}$ & $8.00 \mathrm{~b}$ & $0.63 \mathrm{~b}$ & 2.33 \\
\hline P37 & 7.00 & $1.06 \mathrm{ab}$ & $16.00 \mathrm{abc}$ & $0.28 \mathrm{abcd}$ & $2.58 \mathrm{ab}$ & $2.67 \mathrm{ab}$ & $13.33 \mathrm{abc}$ & $15.67 a b$ & $1.18 b$ & 2.67 \\
\hline P38 & 7.00 & $1.12 \mathrm{ab}$ & $14.67 \mathrm{abc}$ & $0.35 \mathrm{abcd}$ & $3.25 a b$ & $2.00 a b$ & $18.33 a b c$ & $11.33 b$ & $0.62 \mathrm{~b}$ & 2.33 \\
\hline
\end{tabular}


Table 1. Continuation

\begin{tabular}{|c|c|c|c|c|c|c|c|c|c|c|}
\hline Genotype & $\begin{array}{l}\text { Stem } \\
\text { diameter }^{1} \\
(\mathrm{~mm})\end{array}$ & $\begin{array}{l}\text { Plant } \\
\text { height } \\
\text { (m) }\end{array}$ & $\begin{array}{l}\text { Secondary } \\
\text { branches } \\
\text { number }\end{array}$ & $\begin{array}{l}\text { Shoot } \\
\text { biomass per } \\
\text { plant } \\
(\mathrm{kg})\end{array}$ & $\begin{array}{l}\text { Root } \\
\text { yield per plant } \\
(\mathrm{kg})\end{array}$ & $\begin{array}{l}\text { Number of } \\
\text { roots per } \\
\text { plant }\end{array}$ & $\begin{array}{l}\text { Root } \\
\text { diameter (D) } \\
(\mathrm{cm})\end{array}$ & $\begin{array}{l}\text { Root } \\
\text { length }(\mathrm{L}) \\
(\mathrm{cm})\end{array}$ & $\mathrm{L} / \mathrm{D}$ ratio & $\begin{array}{l}\text { Root } \\
\text { shape }\end{array}$ \\
\hline P39 & 7.67 & $0.65 a b$ & $17.00 \mathrm{abc}$ & $0.25 \mathrm{abcd}$ & $3.14 a b$ & $2.00 \mathrm{ab}$ & $18.67 \mathrm{abc}$ & $12.33 \mathrm{ab}$ & $0.66 \mathrm{~b}$ & 2.33 \\
\hline P39 & 7.67 & $0.65 a b$ & $17.00 \mathrm{abc}$ & $0.25 \mathrm{abcd}$ & $3.14 a b$ & $2.00 a b$ & $18.67 \mathrm{abc}$ & $12.33 \mathrm{ab}$ & $0.66 \mathrm{~b}$ & 2.33 \\
\hline P40 & 9.67 & $1.00 \mathrm{ab}$ & $19.33 a b c$ & $0.44 \mathrm{abcd}$ & $2.95 \mathrm{ab}$ & $2.33 a b$ & $17.33 \mathrm{abc}$ & $9.33 \mathrm{~b}$ & $0.52 \mathrm{~b}$ & 1.67 \\
\hline P41 & 8.33 & $0.94 a b$ & $15.67 \mathrm{abc}$ & $0.32 \mathrm{abcd}$ & $2.37 \mathrm{ab}$ & $2.33 a b$ & $18.33 a b c$ & $7.67 \mathrm{~b}$ & $0.41 \mathrm{~b}$ & 1.67 \\
\hline P42 & 8.00 & $1.15 \mathrm{ab}$ & $14.00 a b c$ & $0.55 \mathrm{abcd}$ & $3.47 \mathrm{ab}$ & $3.00 \mathrm{ab}$ & $18.33 \mathrm{abc}$ & $11.33 \mathrm{~b}$ & $0.66 \mathrm{~b}$ & 2.33 \\
\hline P43 & 4.67 & $1.00 \mathrm{ab}$ & $22.33 a b c$ & $0.63 \mathrm{abcd}$ & $2.49 a b$ & $3.33 a b$ & $15.00 a b c$ & $12.00 \mathrm{ab}$ & $0.81 b$ & 3.00 \\
\hline P44 & 9.67 & $1.33 \mathrm{ab}$ & $30.67 \mathrm{a}$ & $0.79 a b c$ & $4.09 \mathrm{ab}$ & $2.33 \mathrm{ab}$ & $18.67 \mathrm{abc}$ & $14.67 \mathrm{ab}$ & $0.87 \mathrm{~b}$ & 2.33 \\
\hline P45 & 6.67 & $0.98 a b$ & $21.00 a b c$ & $0.51 \mathrm{abcd}$ & $2.95 \mathrm{ab}$ & $2.00 a b$ & $17.67 \mathrm{abc}$ & $11.33 b$ & $0.84 \mathrm{~b}$ & 2.33 \\
\hline P46 & 8.00 & $0.83 \mathrm{ab}$ & 13.33 abc & $0.43 \mathrm{abcd}$ & $2.79 a b$ & $2.33 \mathrm{ab}$ & $17.00 \mathrm{abc}$ & $12.00 \mathrm{ab}$ & $0.91 \mathrm{~b}$ & 3.00 \\
\hline P47 & 5.00 & $0.82 a b$ & $14.00 \mathrm{abc}$ & $0.31 \mathrm{abcd}$ & $2.64 \mathrm{ab}$ & $2.00 a b$ & $16.67 a b c$ & $10.67 \mathrm{~b}$ & $0.65 \mathrm{~b}$ & 1.67 \\
\hline P48 & 8.67 & $1.62 \mathrm{a}$ & 18.33 abc & $0.37 \mathrm{abcd}$ & $3.07 \mathrm{ab}$ & $2.33 \mathrm{ab}$ & $16.33 a b c$ & $11.00 \mathrm{~b}$ & $0.68 \mathrm{~b}$ & 2.67 \\
\hline P49 & 7.67 & $0.98 a b$ & 11.66 bc & $0.26 \mathrm{abcd}$ & $2.29 \mathrm{ab}$ & $2.33 a b$ & $15.67 a b c$ & $12.00 a b$ & $0.88 \mathrm{~b}$ & 2.00 \\
\hline P50 & 6.33 & $0.88 \mathrm{ab}$ & $14.00 \mathrm{abc}$ & $0.24 \mathrm{abcd}$ & $3.39 a b$ & $2.33 \mathrm{ab}$ & $16.33 a b c$ & $11.00 \mathrm{~b}$ & $0.67 \mathrm{~b}$ & 2.67 \\
\hline P51 & 7.67 & $1.38 \mathrm{ab}$ & $13.33 a b c$ & $0.32 \mathrm{abcd}$ & $4.43 a b$ & $2.33 \mathrm{ab}$ & $20.00 \mathrm{ab}$ & $9.67 \mathrm{~b}$ & $0.49 \mathrm{~b}$ & 1.67 \\
\hline P52 & 5.33 & $0.75 a b$ & $8.33 \mathrm{c}$ & $0.14 \mathrm{bcd}$ & $2.32 \mathrm{ab}$ & $4.33 \mathrm{a}$ & $16.33 a b c$ & $15.00 \mathrm{ab}$ & $0.95 \mathrm{~b}$ & 2.67 \\
\hline P53 & 7.00 & $0.58 \mathrm{ab}$ & $16.00 \mathrm{abc}$ & $0.23 \mathrm{abcd}$ & $2.39 \mathrm{ab}$ & $2.67 \mathrm{ab}$ & $14.33 \mathrm{abc}$ & $12.33 a b$ & $0.88 \mathrm{~b}$ & 1.67 \\
\hline P54 & 6.00 & $0.78 \mathrm{ab}$ & $11.33 \mathrm{bc}$ & $0.14 \mathrm{bcd}$ & $1.70 \mathrm{~b}$ & $2.33 a b$ & $13.67 \mathrm{abc}$ & $9.67 \mathrm{~b}$ & $0.71 \mathrm{~b}$ & 2.67 \\
\hline P55 & 8.33 & $1.12 a b$ & $26.67 \mathrm{ab}$ & $0.80 \mathrm{ab}$ & $2.58 \mathrm{ab}$ & $3.67 \mathrm{ab}$ & $17.67 \mathrm{abc}$ & $11.00 \mathrm{~b}$ & $0.60 \mathrm{~b}$ & 2.00 \\
\hline P56 & 7.00 & $0.70 a b$ & $14.00 \mathrm{abc}$ & $0.17 \mathrm{abcd}$ & $2.80 \mathrm{ab}$ & $2.00 a b$ & $16.00 \mathrm{abc}$ & $13.33 \mathrm{ab}$ & $0.81 \mathrm{~b}$ & 3.00 \\
\hline P57 & 8.33 & $0.61 \mathrm{ab}$ & $13.67 \mathrm{abc}$ & $0.40 \mathrm{abcd}$ & $1.96 \mathrm{ab}$ & $1.33 \mathrm{~b}$ & $16.33 a b c$ & $11.33 b$ & $0.69 \mathrm{~b}$ & 1.67 \\
\hline P58 & 7.33 & $1.00 \mathrm{ab}$ & $14.33 a b c$ & $0.34 \mathrm{abcd}$ & $2.85 a b$ & $2.67 a b$ & $15.00 \mathrm{abc}$ & $10.00 \mathrm{~b}$ & $0.69 \mathrm{~b}$ & 3.00 \\
\hline P59 & 8.33 & $1.09 a b$ & $16.00 \mathrm{abc}$ & $0.51 \mathrm{abcd}$ & $2.63 \mathrm{ab}$ & $2.67 a b$ & $13.33 a b c$ & $12.00 \mathrm{ab}$ & $1.08 \mathrm{~b}$ & 2.67 \\
\hline P60 & 7.00 & $1.16 a b$ & 12.33 bc & $0.36 \mathrm{abcd}$ & $1.91 \mathrm{ab}$ & $2.33 \mathrm{ab}$ & $13.33 a b c$ & $10.33 \mathrm{~b}$ & $0.75 \mathrm{~b}$ & 2.67 \\
\hline P61 & 7.00 & $0.59 a b$ & $9.67 \mathrm{bc}$ & $0.12 \mathrm{~d}$ & $1.48 b$ & $1.33 \mathrm{~b}$ & $9.67 \mathrm{c}$ & $10.67 \mathrm{~b}$ & $1.25 \mathrm{~b}$ & 1.67 \\
\hline P62 & 8.00 & $0.80 \mathrm{ab}$ & $12.33 \mathrm{bc}$ & $0.29 \mathrm{abcd}$ & $1.89 \mathrm{ab}$ & $2.00 \mathrm{ab}$ & $15.33 \mathrm{abc}$ & $11.33 \mathrm{~b}$ & $0.72 \mathrm{~b}$ & 2.33 \\
\hline P63 & 8.00 & $0.79 a b$ & $15.67 a b c$ & $0.33 \mathrm{abcd}$ & $1.88 \mathrm{ab}$ & $2.67 \mathrm{ab}$ & $13.33 a b c$ & $8.67 \mathrm{~b}$ & $0.66 \mathrm{~b}$ & 3.00 \\
\hline P64 & 6.67 & $1.09 a b$ & $11.00 \mathrm{bc}$ & $0.45 \mathrm{abcd}$ & $2.69 \mathrm{ab}$ & $2.00 \mathrm{ab}$ & $17.33 \mathrm{abc}$ & $9.67 \mathrm{~b}$ & $0.56 \mathrm{~b}$ & 2.67 \\
\hline Mean & 7.26 & 0.91 & 14.93 & 0.34 & 2.78 & 2.46 & 16.17 & 12.50 & 0.87 & 2.39 \\
\hline CV \% & 37.20 & 45.71 & 45.81 & 72.62 & 48.21 & 39.62 & 23.56 & 36.87 & 47.54 & 35.07 \\
\hline
\end{tabular}

${ }^{1}$ This character had not significant difference between means. ${ }^{2}$ Visual assessment means followed by the same letter did not differ at the Duncan test $(P \leq 0.05) \mathrm{CV}-$ Coefficient of variation

a perpendicular line was drawn on the middle of the root yield vector (Figure 2), which distinguished P44, P22, P18, P55 and P17. In addition, these genotypes tended to have a high number of secondary branches, high shoot biomass, root diameter and plant height values. In contrast, this figure also shows lower values for P61, P36, P11, P54 and P15 genotypesassociated characters. On the other hand, accessions P46, P33 cannot be distinguished with these traits; they can be observed near the vector origin, coordinate $(0,0)$ (Figure 1).
Biplot also shows trait contribution to ascertain the diversity, which is related to vector norm. The major traits were tuber yield, number of secondary branches, shoot biomass, plant height, root length and root diameter. Root yield presented variability from 1.34 to $5.38 \mathrm{~kg}$ per plant. P22 accession had highest root yield, which is equivalent to 107.6 t ha ${ }^{-1}$. P51, P6, P18, P17 and P44 genotypes yielded above 4 $\mathrm{kg}$ per plant; which is equivalent to more than $80 \mathrm{t} \mathrm{ha}^{-1}$. The lowest root yield was equivalent to $27 \mathrm{t} \mathrm{ha}^{-1}$ corresponding to P57 and P61 genotypes. 


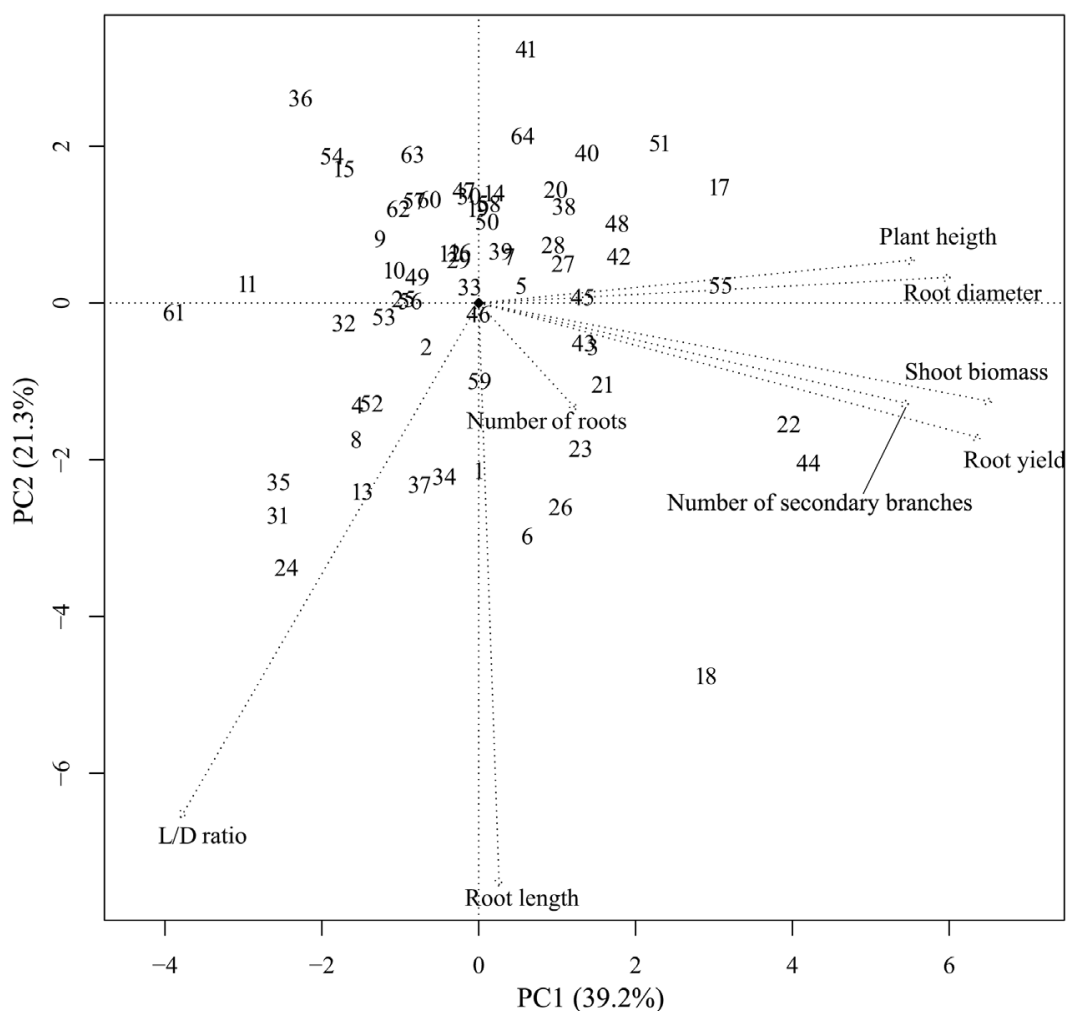

Figure 1. Biplot visualizing genetic diversity of 64 genotypes assessed through 8 morphoagronomic characters.

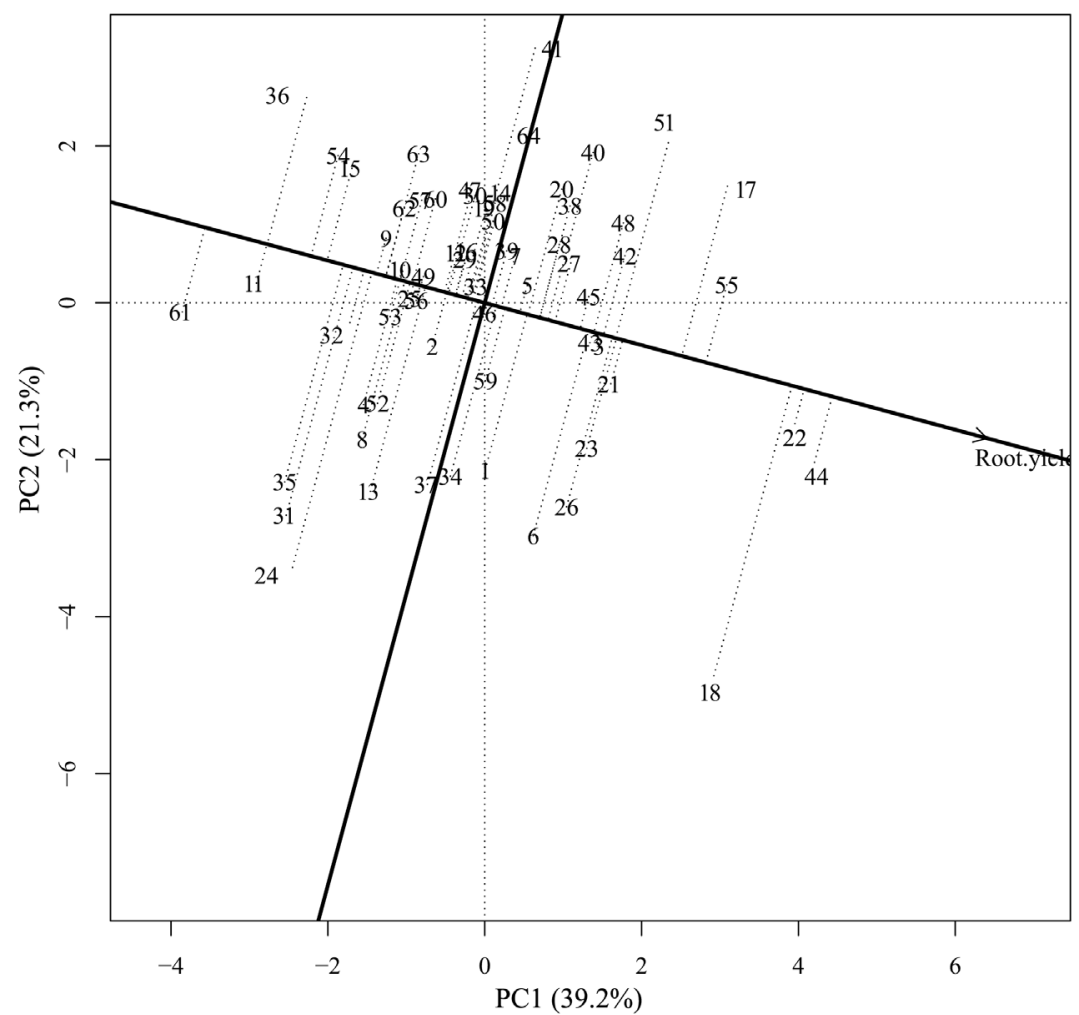

Figure 2. Biplot shows tuber yield vector associated with 64 yam bean genotypes. 
UPGMA cluster analysis showed 15 major groups (Figure 3 ), at $50 \%$ of relative distance. The most distinct groups presented one genotype per group, which were P18, P22, P44, P55, P61 and P52. P18 and P44 genotypes were more promising on account of their elevated tuber yield and large number of secondary branches.

Visually assessed root shape show non-significant variability by ANOVA (Table 1). Consequently Length/ Diameter ratio is recommended for accurate evaluation. Root shape ranged from globular to flat. Only the accession P26 had a very elongated root; its length/diameter ratio was 4 to 1. Ribeiro et al. (2011) found in P26 globular, flat and elongated roots. To know the main format will be necessary perform experiments with larger numbers of plants per plot.

This study was carried out with relative small plots with three plants; nevertheless, there was no plants loss. These results suggest that there are diversity of yam bean genotypes and potential genotypes to cultivate.

\section{DISCUSSION}

Morphological diversity analyses in Pachyrhizus spp. showed the ability to discriminate three cultivated species (Zanklan 2003) and groups within P. tuberosus (Tapia and Sorensen 2003) as 'ashipas', 'chuins' and 'jíquimas'.

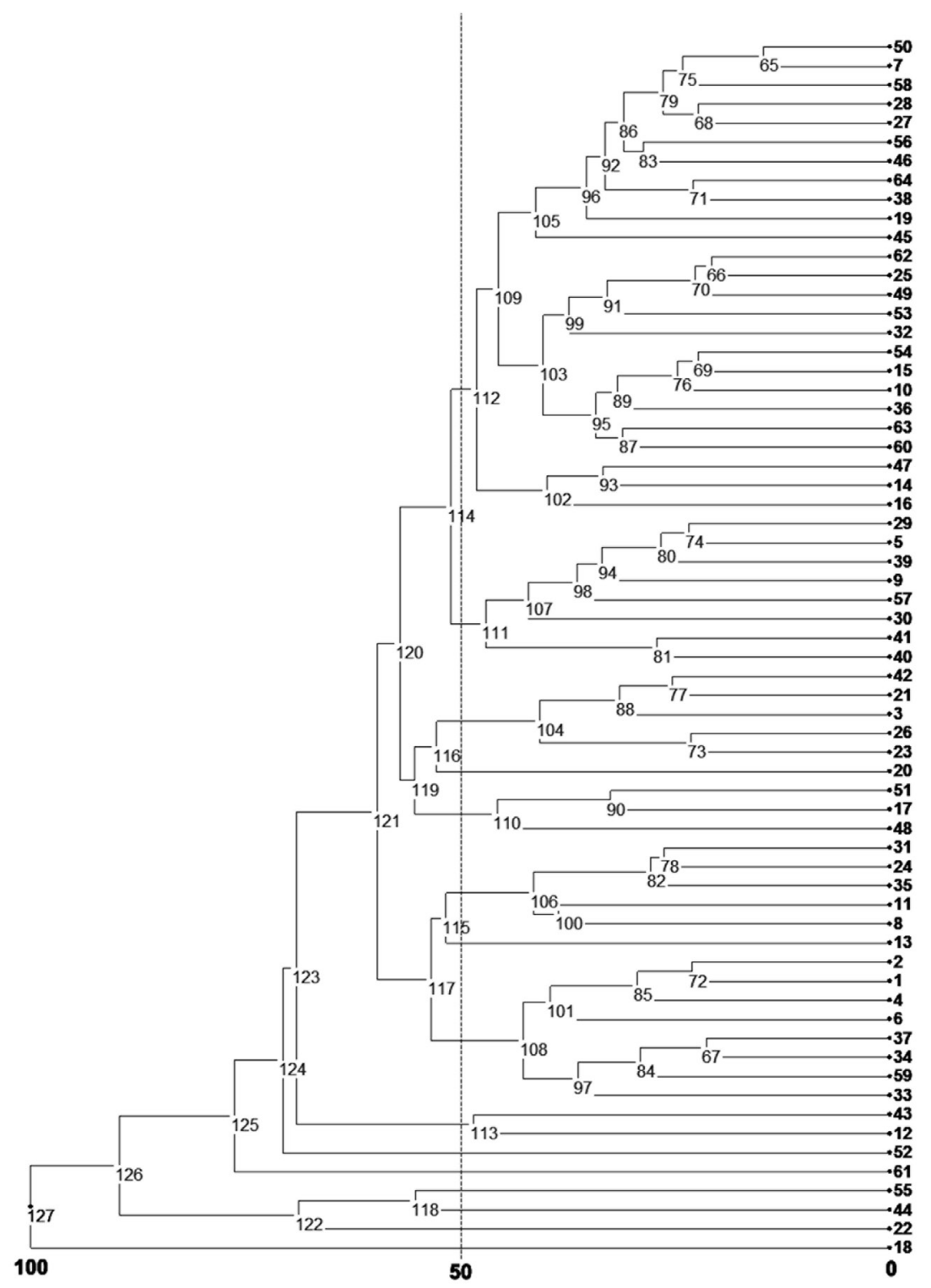

Figure 3. Dendrogram for 64 yam bean genotypes based on Euclidean distances calculated through 1000 bootstraps and UPGMA (Unweighted pair group method with arithmetic mean). 
This indicates the ability of morphological characters to discriminate genotypes in yam bean.

Diversity analyses performed elsewhere (Delêtre et al. 2013; Santayana et al. 2014; Tapia and Sorensen 2003) included few materials from Brazil. Therefore, Brazilian diversity needs to be ascertained. Likewise the former studies on these genotypes did not focus on diversity (Noda 1991; Ribeiro et al. 2011).

We found all characters had high diversity, the exception was stem diameter and root shape. Although, both characters ranged from $4.7-10.3 \mathrm{~mm}$ and $1.3-3.0$ respectively, their variation was not significant, due to importance of the environment effect with genetic effect tending to be null. Furthermore, visual assessment of root shape was not efficient when compared with its length/diameter ratio value. Thus, quantitative methods show to be better than visual ones.

We found genotypes presented high yield, above $80 \mathrm{tha}^{-1}$. These 64 genotypes have been studied twice using the same spacing, season and local used in this research (Noda 1991; Ribeiro et al. 2011). Noda (1991) reported root yield up to 50 t ha ${ }^{-1}$. Ribeiro et al. (2011) found $44 \mathrm{t} \mathrm{ha}^{-1}$ as maximum root yield in genotypes P51 and P54; the lowest root yield ranged from 3.1 to $5.4 \mathrm{tha}^{-1}$ in P1, P14, P17 and P61 genotypes.

Those previous studies identified the P51 genotype as the more stable and adapted, with respect to high tuber yield in non-flooded land. Regarding tuber yield under non-flooded land P61 is a genotype with low adaption. Therefore, P51 must be tested in other environments to confirm its stability. On the other hand, there is a notable difference of tuber yield values in our study when compared to those of Noda (1991) and Ribeiro et al. (2011). In our study the maximum tuber yield was twice that of those studies. This can be explained by year $\mathrm{x}$ genotype interaction.

Root length and L/D ratio diversity point out that there is root shape variation, highlighting P18 with the highest roots length $(23 \mathrm{~cm})$ and P26 with the highest root L/D ratio (4.2). This fact indicates P18 as a more promising genotype due to its high tuber yield and large size.

Biplot analysis shows diversity and relation between characters (Figure 1). Yam bean genotypes are distributed in the four quadrants, indicating their wide diversity formerly detected by Duncan test.

Considering only these morphological characters they can be grouped in 3 groups. Group 1: Plant height, root diameter, shoot biomass, number of secondary branches and root yield. Group 2: Number of roots. Group 3: Root length/diameter ratio and length. In general, Group 1 and Group 3 vectors tend to pertain to an orthogonal relationship (90 degrees angle). This indicates these groups are independent from each other. Group 2 or number of roots shows an intermediate behavior to discriminate diversity since all genotypes bear multituberous roots. In addition, this character presents low variance, which is represented by smaller vector norm. In Group 1 characters can be associated physiologically, on account of the large number of secondary branch and high shoot biomass, which can increase photosynthesis leading to translocation of photoassimilates to the roots (Savage et al. 2013).

However, other studies found different discriminant traits in Pachyrhizus. For instance Tapia and Sorensen (2003), using D ratio/index, found that outline of terminal leaflet, pulp color of root and seed shape are major discriminant traits between groups of $P$. tuberosus (Ashipa, Chuin, Jíquima and Ashipa hybrids). Sorensen et al. (1997) with three principal components (61\% of total variation) showed that major discriminant traits are petal characters, petioles, petiolules, length/diameter (L/D) ratio of lateral and terminal leafleats. Although, Tapia and Sorensen (2003) considered root traits such as L/D ratio and tuber yield per plant, they were not useful to discriminate between groups. Probably each group has similar root yield variability. Sorensen et al. (1997) did not considered root yield traits in 31 genotypes diversity analysis, but they did intercrossings between $P$. tuberosus groups and other species, which resulted in low yielding and undesirable shape roots. Therefore, diversity analysis for breeding objectives must include agronomic traits.

Our findings indicate that a breeding program can be successfully to enhance seed and root yield. Improved varieties can help to recuperate degraded areas and may be another option for Amazonian farmers.

To understand the genetic structure and to identify species of our genotype collection more studies will be need, especially using molecular markers. On the other hand, to select potential genotypes other studies must be done, such as to assess seed and root yield without pruning flowers, as well as, quantifying rotenone and pachyrhizin in seeds. Studies of genotype $\mathrm{x}$ environment interaction also will help to select genotypes adapted to floodplain, non-flooding land and degraded areas.

\section{CONCLUSIONS}

To assess genetic diversity of yam bean one must use plant height, number of secondary branches, shoot biomass, root biomass, number, length, diameter and, length/diameter ratio. There is a wide and valuable diversity of yam bean genotypes, where at least 15 groups were found. At the same time, we found diversity for tuber yield from 27 to $108 \mathrm{tha}^{-1}$. Thus, new varieties can be obtained by selection in a short time. We expect to be able to test promising genotypes in flooded land to select material for family farming in the Amazonian region.

\section{ACKNOWLEDGEMENTS}

We thank to INPA for support of this Project. We also thank to Fundação de Apoio à Pesquisa do Amazonas (FAPEAM) for a Master's scholarship to first author. 


\section{REFERENCES}

Antonio, I. C. 2006. Boletim agrometeorológico 2000: Estação agroclimatológica da Embrapa Amazônia Ocidental, Km 29 da Rodovia AM 010. ed. Manaus, EMBRAPA, 32p.

Bernal, E. F.; Villardon, P. G. 2015. Package 'GGEBiplotGUI'.

Catteau, L.; Lautie, E.; Kone, O.; Coppee, M.; Hell, K.; Pomalegni, C. B. et al. 2013. Degradation of Rotenone in Yam Bean Seeds (Pachyrhizus sp.) through Food Processing. Journal of Agricultural and Food Chemistry, 61: 11173-11179.

Chavez, A. L.; Bedoya, J. M.; Sánchez, T.; Iglesias, C.; Ceballos, H.; Roca, W. 2000. Iron, carotene, and ascorbic acid in cassava roots and leaves. Food Nutrition Bulletin, 21: 410-413.

Delêtre, M.; Soengas, B.; Utge, J.; Lambourdière, J.; Sørensen, M. 2013. Microsatellite Markers for the Yam Bean Pachyrhizus (Fabaceae). Applications in Plant Sciences, 1: 1200551.

Estrella-Parra, E. A.; Gomez-Verjan, J. C.; Gonzalez-Sanchez, I.; Vazquez-Martinez, E. R.; Vergara-Castaneda, E.; Cerbon, M. A. et al. 2014. Rotenone isolated from Pachyrhizus erosus displays cytotoxicity and genotoxicity in K562 cells. Natural Product Research, 28: 1780-1785.

Heider, B.; Tumwegamire, S.; Tukamuhabwa, P.; Ndirigwe, J.; Bouwe, G.; Bararyenya, A. et al. 2011. Nutritional improvement of yam bean and sustainability of farming systems in Central and West Africa. African Crop Science, 10: 93-95.

Kinupp, V. F.; Lorenzi, H. 2014. Plantas Alimentícias Não Convencionais (PANC) no Brasil: guia de identificação, aspectos nutricionais e receitas ilustradas. ed. São Paulo, Instituto Plantarum de Estudos da Flora, 768p.

Lautié, E.; Rozet, E.; Hubert, P.; Vandelaer, N.; Billard, F.; Zum Felde, T. et al. 2013. Fast method for the simultaneous quantification of toxic polyphenols applied to the selection of genotypes of yam bean (Pachyrhizus sp.) seeds. Talanta, 117: 94-101.

Leuner, O.; Havlik, J.; Budesinsky, M.; Vrkoslav, V.; Chu, J.; Bradshaw, T. D. et al. 2013. Cytotoxic Constituents of Pachyrhizus tuberosus from Peruvian Amazon. Natural Product Communications, 8: 1423-1426.

Nielsen, P. E.; Sørensen, M.; Halafihi, M. 2000. Yield potential of Yam Bean (Pachyrhizus erosus (L.) Urban) accessions in the Kingdom of Tonga, South Pacific. Tropical Agriculture, 77: 174-179.

Noda, H.1991. Potencialidade da cultura de feijão macuco (Pachyrhizus tuberosus). In: SUFRAMA (Ed.). Introdução à Horticultura e fruticultura no Amazonas. Manaus: CNPq/INPASUFRAMA, p.58-65.

Noda, H.; Kerr, W. E. 1983. The effects of staking and of pruning on the root production of yam bean (Pachyrrhizus tuberosus). Tropical Grain Legume Bulletin, 27: 35-37.
Padonou, S. W.; Houyèvou, A. K.; Ahounou, J. L.; Houssou, A. P.; Fandohan, P.; Aïhou, K. et al. 2013. Yam bean (Pachyrhizus erosus) tuber pro-cessing in Benin: production and evaluation of the quality of yam bean-gari and yam bean-fortified gari. International Journal of Biology and Chemistry Science, 7: 247259.

Perrier, X.; Jacquemoud-Collet, J. P. 2006. DARwin software.

R Core Team. 2015. $R$ : A language and environment for statistical computing. Vienna, R Foundation for Statistical Computing, http://www.R-project.org.

Ribeiro, W. G.; Noda, H.; Soares, J. E. C.; Rocha, M. Q. 2011. Avaliação das caracteristicas agronomicas de feijão-macuco. Horticultura Brasileira, 29: s2902-s2908.

Santayana, M.; Rossel, G.; Núñez, J.; Sørensen, M.; Delêtre, M.; Robles, R. et al. 2014. Molecular Characterization of Cultivated Species of the Genus Pachyrhizus Rich. ex DC. by AFLP Markers: Calling for More Data. Tropical Plant Biology, 7: 121-132.

Savage, J. A.; Zwieniecki, M. A.; Holbrook, N. M. 2013. Phloem transport velocity varies over time and among vascular bundles during early cucumber seedling development. Plant Physiology, 163: 1409-18.

Sørensen, M. 1996. Yam bean (Pachyrhizus DC.). In: Heller et al. (Ed.), Vol 2. Promoting the conservation and use of underutilized and neglected crop, Institute of Plant Genetics and Crop Plant Research . Rome: International Plant Genetic Resources Institute, p.1-141.

Sorensen, M.; Doygaard, S.; Estrella, J.; Kvist, L.; Nielsen, P. 1997. Status of the South American tuberous legume Pachyrhizus tuberosus (Lam.) Spreng.: Field observations, taxonomic analysis, linguistic studies and agronomic data on the diversity of the South American Pachyrhizus tuberosus (Lam.) Spreng. complex with special reference to the identification of two new cultivar groups from Ecuador and Peru. Biodiversity and Conservation, 6: $1581-1625$.

Tapia, C.; Sorensen, M. 2003. Morphological characterization of the genetic variation existing in a Neotropical collection of yam bean, Pachyrhizus tuberosus (Lam.) Spreng. Genetic Resources and Crop Evolution, 50: 681-692.

Zanklan, A. S. 2003. Agronomic performance and genetic diversity of the root crop yam bean (Pachyrhizus spp.) under West African conditions. PhD Doctor. Faculty of Agricultural Sciences, GeorgAugust University, Göttingen. 123p.

Zanklan, A. S.; Ahouangonou, S.; Becker, H. C.; Pawelzik, E.; Grueneberg, W. J. 2007. Evaluation of the storage rootforming legume yam bean (Pachyrhizus spp.) under west African conditions. Crop Science, 47: 1934-1946.

Recebido em 23/10/2015

Aceito em 04/01/2016 